\title{
Surface Area Estimation of Digitized 3D Objects Using Local Computations
}

\author{
Joakim Lindblad and Ingela Nyström \\ Centre for Image Analysis, Uppsala University \\ Lägerhyddvägen 17, SE-75237 Uppsala, SWEDEN \\ joakim, ingela@cb.uu.se
}

\begin{abstract}
We describe surface area measurements based on local estimates of isosurfaces originating from a marching cubes representation. We show how improved precision and accuracy are obtained by optimizing the area contribution for one of the cases in this representation. The computations are performed on large sets (approximately 200,000 $3 \mathrm{D}$ objects) of computer generated spheres, cubes, and cylinders. The synthetic objects are generated over a continuous range of sizes with randomized alignment in the digitization grid. Sphericity, a scale invariant measure of compactness, allows us, in combination with the improved surface area estimate, to distinguish among the test sets.
\end{abstract}

Keywords: shape analysis, marching cubes, isoperimetric inequality, accuracy, rotation invariance

\section{Introduction}

In quantitative shape analysis, it is important to know the precision and accuracy of the measurement, and if there are any restrictions on the input domain where the reliability of the measure does not hold. One aim of this paper was to study how $3 \mathrm{D}$ shape can be measured in terms of compactness. In 2D image analysis, $P^{2} / A$ is a commonly used measure of compactness which may be derived from the classical isoperimetric inequality [2]. An "inverse" to compactness is the circularity of objects. This concept is extendable to higher dimensions. Hence, in $3 \mathrm{D}$ image analysis, one feature of interest when distinguishing among different classes of objects is sphericity. We define this as a dimensionless ratio between enclosed volume and surface area.

The problem that arises is that, in image analysis, we are given only a digitized version of the original continuous object. Under these conditions how reliable is our measure? Note that there is a difference in measuring digital objects and measuring digitized objects. The former class of objects exists solely in the digital world, and exact measures can be calculated. The latter represents the digitization of continuous original objects. The aim of the measure is not to find properties of the digital version, but rather to estimate properties of the continuous original. in the methods applied. Also, the measurement on the digitized

A. Braquelaire, J.-O. Lachaud, and A. Vialard (Eds.): DGCI 2002, LNCS 2301, pp. 267-278 2002. (C) Springer-Verlag Berlin Heidelberg 2002 
object can never become more than an estimate, as information is irreversibly lost in the digitization process.

This paper focuses on how the surface area can be measured by an approximating isosurface generated by the Marching Cubes approach [13]. Our aim is to find a way to obtain accurate estimates with high precision based on local computations, avoiding strong assumptions about the object of study. We also verify the robustness of the sphericity measurement for small to large convex objects with randomized alignment in the digitization process.

\section{Surface Area of 3D Objects}

In 2D image analysis, perimeter has been, and sometimes still is, measured in terms of cumulative distance from pixel centre to pixel centre. This is straightforward to accomplish using the Freeman chain code [5], but results in incorrect measurements. Note that the weights 1 for horizontal and vertical steps and $\sqrt{2}$ for diagonal steps are not optimal when measuring digitized line segments 6. By assigning optimized weights [1713] to the steps, more accurate perimeter measurements are obtained. If the boundary is represented by the pixel edges between object and background, steps can be taken from pixel edge to pixel edge, taking advantage of optimized weights, with promising results [12. This corresponds to Marching Squares, a 2D equivalent of Marching Cubes.

In 3D image analysis, when measuring surface area, an analogous first approach would be to connect voxel centre to voxel centre and add the resulting areas. This is not simple and would, as in the 2D case, produce incorrect measurements. In a second approach, where the surface is represented by the faces of the voxels at the boundary between object and background [15, the number of faces gives an efficient and simple estimation of the surface area, but this is an overestimate [14]. By approximating the boundary between object and background with a triangular representation, e.g., the one obtained from the marching cubes algorithm, more correct surface area estimates are obtained.

There exist recent publications [79] that have studied the problem of surface area estimation. Our approach is different in that we exploit the simplicity of working in small neighbourhoods and base our estimates on local computations, still sacrificing neither precision nor accuracy.

\section{Triangular Isosurface Representation}

The voxel representation of object and background has a close relation to polygonal representations; a digital surface can be transformed directly into a triangulated isosurface [10[11]. An $m$-cube (short for Marching Cube), is the cube bounded by the eight voxels in a $2 \times 2 \times 2$ neighbourhood. Hence, each corner of the $m$-cube corresponds to a voxel.

If object and background are considered, the possible number of configurations of the eight voxels is 256 . Each configuration consists of zero to four 


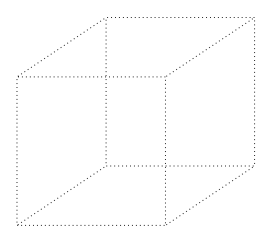

(a) Case 0

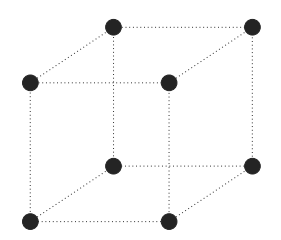

(b) Case 0

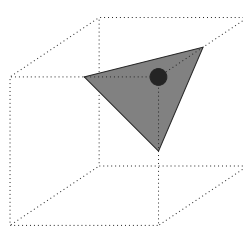

(c) Case 1

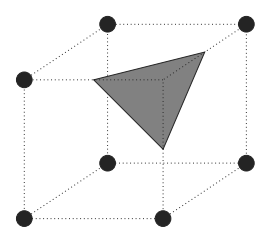

(d) Case 1

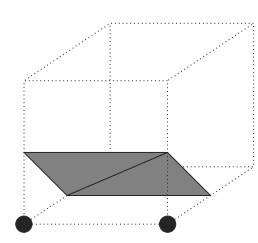

(e) Case 2

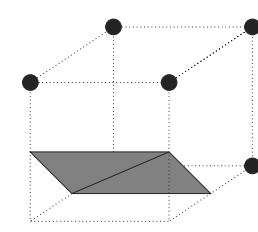

(f) Case 2

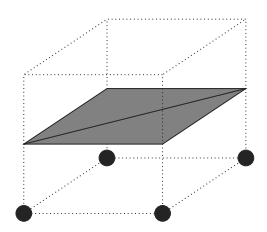

(g) Case 8

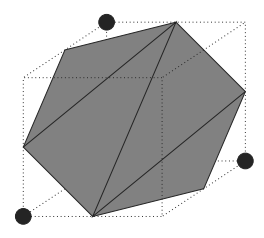

(h) Case 9

Fig. 1. $m$-cubes of $2 \times 2 \times 2$ voxels, where voxels denoted by a $\bullet$ are inside the object and the other voxels are outside. The isosurfaces for the configurations consist of zero to four triangles.

triangles constituting the isosurface through the specific $m$-cube. The configurations are often grouped into symmetry and complementary cases, resulting in 14 (or 15) cases [1316]. See Figures 1 and 2 for examples. Two of these configurations do not represent a boundary situation and have no triangles. These correspond to the case when the $m$-cube is placed completely outside or completely inside the object (Figures 1(a) and 1(b), respectively). We number the cases according to previous literature [13], except for cases 11 and 14 which we group into one case 11. Some of the cases can be triangulated in different ways, e.g., case 11, which is illustrated in Figures 2(a) and 2(b) We use the more symmetric triangulation in Figure 2(b).

The approximated isosurface in the $m$-cube is computed from some interpolation of the voxel values. The interpolation results in intersection points on the edges of the $m$-cube for the triangle vertices. In the following, we will use the simplest case, where the intersection points are positioned midway along the $m$-cube edges.

The correct connection among intersection points is ambiguous for some configurations of voxels. See Figures 2(c) and 2(d) for an example. This classical problem in the original marching cubes algorithm was pointed out by Dürst in 1988 [4]. The problem requires a careful consideration when a closed surface is desired. It has been examined and solved (e.g., [168]). For this study it should be sufficient to assign a contributing area for each configuration. 


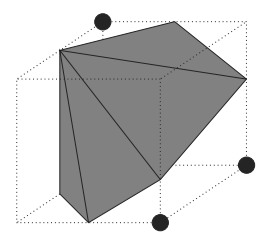

(a) Case 11

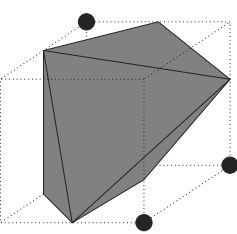

(b) Case 11

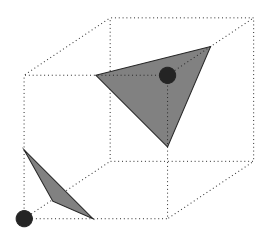

(c) Case 3

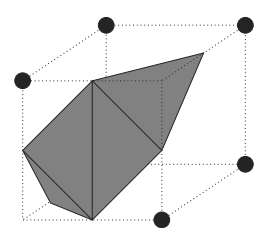

(d) Case 3

Fig. 2. (a), (b): Some $m$-cubes can be triangulated in different ways (vertices moved from the midpoint for visibility reasons). We use the more symmetric version (b). (c), (d): Shown are the triangles we use for these ambiguous $m$-cube configurations.

\section{Sphericity}

In $2 \mathrm{D}$ image analysis, $P^{2} / A$ is a commonly used measure for compactness, or circularity, of objects. The concept behind this dimensionless ratio is extendable to higher dimensions. In 3D image analysis, a feature of interest when distinguishing among different classes of objects would, hence, be the dimensionless ratio of surface area to volume of an object. For a consistent and natural definition of this class of ratios, we choose the following for the 2D case and correspondingly for the 3D case:

Circularity, C: perimeter of a circle, enclosing the same area $A$ as the object, divided by the perimeter $P$ of the object.

$$
\mathbf{C}=\frac{\sqrt{4 \pi A}}{P}
$$

Sphericity, S: surface area of a sphere, enclosing the same volume $V$ as the object, divided by the surface area $A$ of the object.

$$
\mathbf{S}=\frac{\sqrt[3]{36 \pi V^{2}}}{A}
$$

In the continuous case, these measures are in the range $[0,1]$ given by the isoperimetric inequality. The upper bound is reached only by the ball of the corresponding dimension. For the $n \mathrm{D}$ case, the dimension of the ratio is length $h^{n-1} /$ length $^{n-1}$. An alternative would be to define the ratio as length $h^{n} /$ length $^{n}$, but we choose the one formulated above as it more closely reflects the dimension of the predominant part of the measurement 1 The reason for inverting the $P^{2} / A$ expression is that circularity should reach its maximum for a true circle.

\footnotetext{
${ }^{1}$ The measure $P^{2} / A$ suffers from the fact that the perimeter has a more predominant effect on the measure than the area.
} 


\section{Experiments}

We have generated sets of synthetic convex objects. Each set consists of a certain shape digitized in different sizes, rotations, and positions, with respect to the digitization grid. The objects are generated in the continuous space and then discretized using Gauss centre point digitization, where the digitized object is defined to be the set of all grid points (voxel centres) contained in the continuous set. All units are given with respect to the sampling grid, i.e., edge length, face area, and volume of voxels are all equal to 1.

The surface area contribution for each of the 256 marching cube configurations, $A_{M C(i)}, i=0 . .255$, is precomputed by summing the areas of the included triangles and stored in a lookup table [14. The histogram describing the number of each of the 256 configurations, $n_{i}$, is computed for each digitized object. The surface area of the specific object is then calculated as

$$
A=\sum_{i=0}^{255} n_{i} A_{M C(i)}
$$

Of interest is to plot the ratio of estimated area to true area versus the size of the object. The variance within a given size, for different rotations and placement of that object, should capture digitization effects. We are also interested in the sphericity, computed as described in Section 4. In a recent publication, the enclosed volume is computed for the marching cubes representation in an incremental fashion from the surface [14]. How this would affect the sphericity estimate needs further studies. Here, the volume $V$ is computed as a simple voxel count of the object, which is a good estimate for large objects.

\subsection{Bias}

Our main assumption is that, on a local scale, objects are fairly flat. The surface of a large sphere is a good sampling of planes in all directions. Therefore, our first test object is a Euclidean sphere. This also happens to be the object with maximal sphericity. The digitization of a sphere centered at $\left(x_{0}, y_{0}, z_{0}\right) \in \mathbb{R}^{3}$ of radius $r \in \mathbb{R}$ is generated by the following equation

$$
f(x, y, z)=\left\{\begin{array}{ll}
1 & \text { if }\left(x-x_{0}\right)^{2}+\left(y-y_{0}\right)^{2}+\left(z-z_{0}\right)^{2} \leqslant r^{2} \\
0 & \text { otherwise }
\end{array}, \quad(x, y, z) \in \mathbb{Z}^{3}\right.
$$

See Figure 3 for sphere examples, where $r$ is 4, 10, and 25, respectively, presented as renditions of their marching cubes triangulations. Figure 4 shows three surface area estimates for digitized spheres of increasing radius. The function describes the mean value for a given radius and the error bars indicate the corresponding smallest and largest estimates, where the surface area is computed according to Eq. (3). If the surface area is computed with the triangle configuration of Figures 5(a) and 5(b) for case 5 (area contribution of 5 flat $=1.150$ ), this gives the largest overestimate $(8.8 \%)$. From now on, we instead use the triangle 


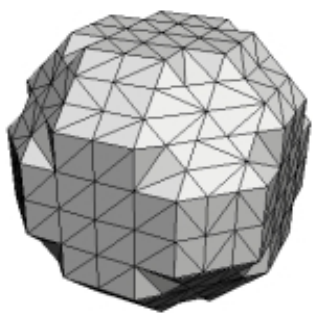

(a) radius 4

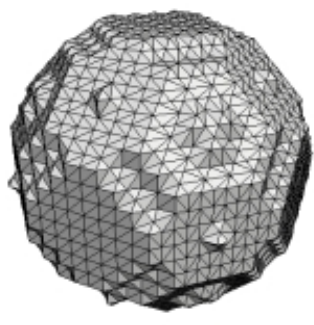

(b) radius 10

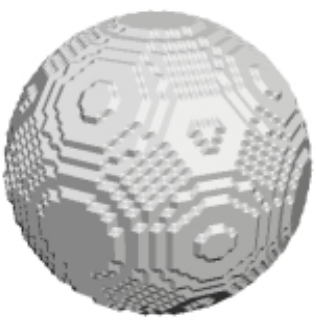

(c) radius 25

Fig. 3. Three spheres presented as renditions of their marching cubes triangulations.

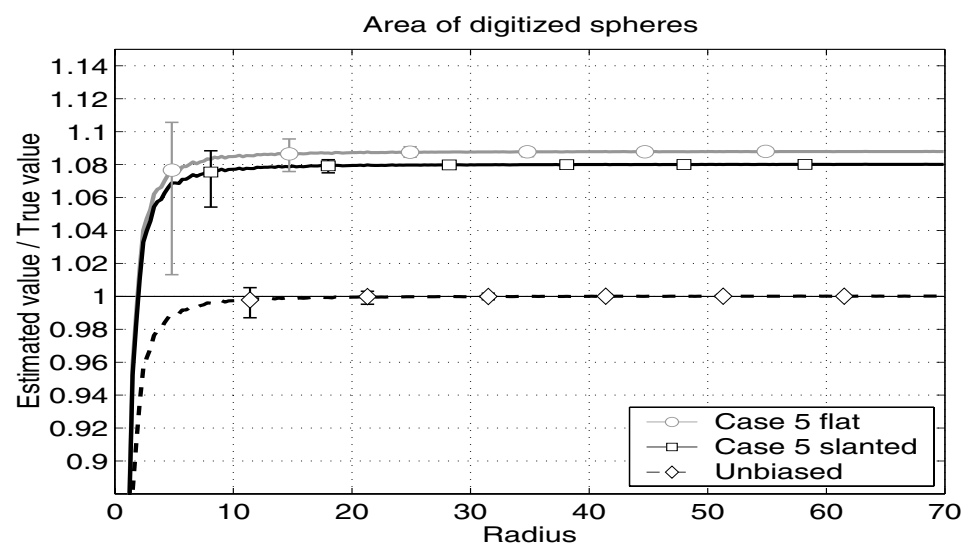

Fig. 4. Three surface area estimates divided by the true surface area, for 27,000 digitized spheres of increasing radius.

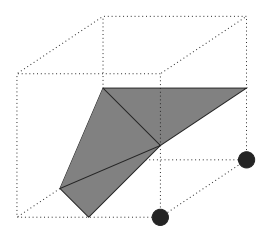

(a) Case 5

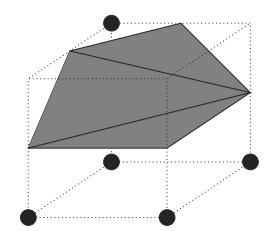

(b) Case 5

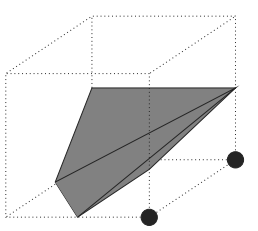

(c) Case 5

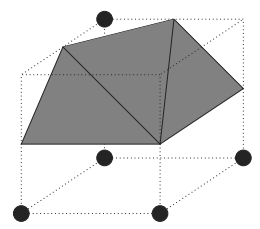

(d) Case 5

Fig. 5. The triangular representations in (a) and (b) may seem as the simplest for this configuration and is possibly the most commonly used. The representations in (c) and (d) approximate a smoother surface. (Third • not visible in (a) and (c).) 


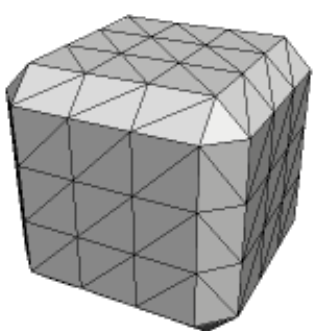

(a) side 4

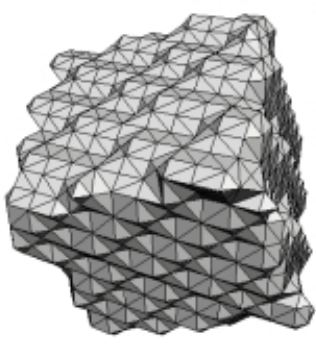

(b) side 10

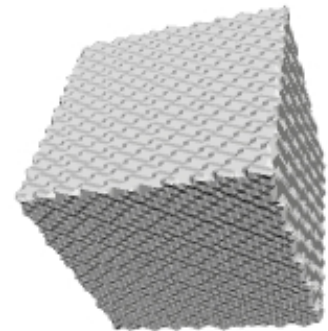

(c) side 40

Fig. 6. Three cubes of different sizes presented as renditions of their marching cubes triangulations. (a) is aligned with the digitization grid. (b) and (c) have the same orientation, rotated $\left(15^{\circ},-20^{\circ}, 25^{\circ}\right)$, but represent different sizes.

configuration of Figures 5(c) and 5(d) for case 5 (area contribution of 5slanted $=1.122$ ), for which the overestimate is slightly reduced $(8.0 \%)$.

To obtain an unbiased estimate for randomly aligned planar surfaces, we choose to divide the area by the average overestimate for large digitized spheres. Using 5slanted the bias term $\xi$ becomes 1.080 (supposed convergence value from Figure 4). This also gives good convergence to the true surface area.

\subsection{Alignment Invariance}

If we wish to study the behaviour of specifically aligned planar surfaces, the sphere is not useful as it represents every alignment. We seek alignment invariance for large planar surfaces, which here is represented by cubes, rotated and positioned in different ways. When applying the same surface area estimator for cubes, the results divided by the bias term $\xi=1.080$ are (on average) an underestimate of the true surface area. This is due to the cutting of corners and edges. The cube in Figure 6(a), aligned with the axes of the digitization grid, is small enough to illustrate this effect. For large objects the effect can be neglected, though.

The area measurements for the cubes also contain a large variance. This is due to the different alignments of the faces of the cube. The surface area becomes too large when the cube is rotated in some direction that is not well captured by the triangles of the marching cubes triangulation, e.g., as in Figures 6(b) and 6(c). The increased variance can be seen in Figure 7 . The approach of a simple scaling by the bias term is not sufficient for good convergence.

We wish to reduce the variance of the estimate. Our approach is to identify the cases for which the area estimate is extreme and to correct for these errors. Therefore, we study the relative frequency of the $m$-cube configurations 


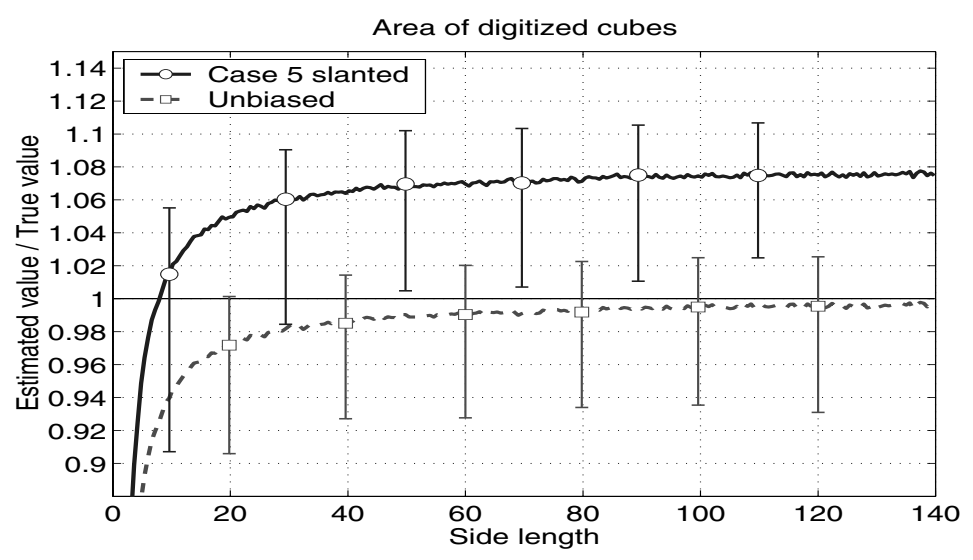

Fig. 7. Two surface area estimates divided by the true surface area, for 80,000 digitized cubes of increasing size.

for cubes of side 100 with different alignment, and examine the situations giving rise to the largest under- and overestimates, respectively. From Figure 8(a), we interpret that some of the $m$-cube configurations, e.g., case 5 , have not been assigned a value agreeing well with the truth. We have already noted two possible triangulations for case 5 (Figure 5), none of which approximates a flat isosurface through the $m$-cube.

By plotting the relative maximum error against a scale factor used on $\mathrm{m}$ cube case 5 (Figure 8(b)), we see that there is an optimum for the scale factor 0.892 . The sum of the three triangle areas of $m$-cube configuration case 5 equals 1.122. If we, instead of assigning this triangle area contribution, assign the value $1.122 * 0.892=1.001$ to case 5 , we reduce the relative maximum error from $7.1 \%$ to $4.3 \%$ and the coefficient of variation (CV, standard deviation divided by mean) from $1.6 \%$ to $1.2 \%$. Note, however, that the scale factor 0.892 is optimal only if case 5 solely is allowed to vary, but the scale factor will change if other cases are also scaled to further improve the surface area estimate.

\subsection{Results}

Our surface area estimator can be summarized in the following: Starting with the surface area from the marching cubes triangulation, we assign to case 5 area contribution 1.001 and divide by the overall bias term $\xi=1.046$. The change of area contribution assigned to case 5 requires a change of the bias term, to acquire an unbiased estimate for randomly aligned planar surfaces as described in Section 5.1 .

To verify our surface area estimates, we will study objects of other shape than spheres and cubes. Here, we choose cylinders with the same height as diameter, a shape resembling the other two, but which still should be possible to distinguish 


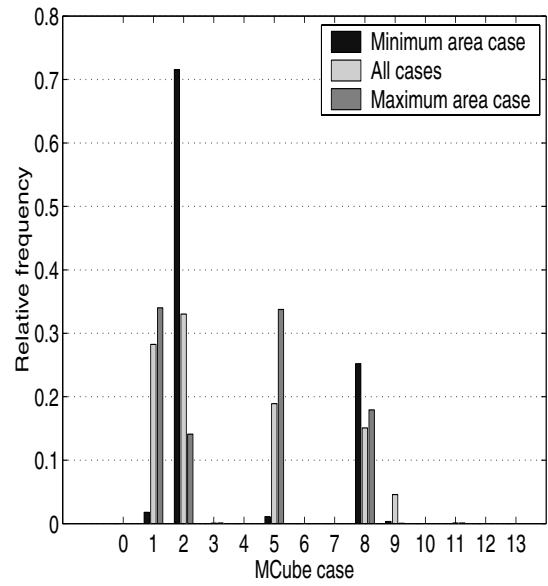

(a)

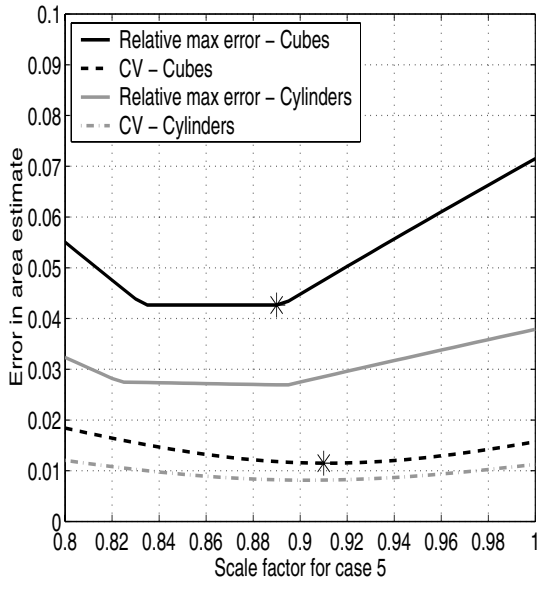

(b)

Fig. 8. (a) Relative frequency of the different $m$-cube cases for 30,000 digitized cubes, of size $100 \times 100 \times 100$, of random position and orientation. (b) Maximum error and coefficient of variation for the estimated area of digitized cubes and cylinders, for different scale factors for the area contribution of $m$-cube case 5 .

as different. As done previously, the cylinders are generated in a continuous range of sizes with randomized alignment in the digitization grid. For the surface area estimate of cylinders (radius 50), the relative maximum error is reduced from $3.8 \%$ to $2.7 \%$, and the CV from $1.1 \%$ to $0.8 \%$ (Figure $8(\mathrm{~b})$ ], using the described method.

Surface area, volume, and sphericity for the three test sets are presented in Figure 9. The plots compare well with expectations. There is still a fair amount of variance in the surface area measurements, but the estimation performance has significantly improved compared to the uncorrected marching cubes area estimate. The volume estimate is robust and show no surprises. The sphericity agrees well, at least for objects down to radius 5, with the sphericity in the continuous case, where a sphere, a cube, and the described cylinder have values $1,0.806$, and 0.874 , respectively. The sphericity measure manages to totally separate the three test sets.

\section{Discussion and Conclusions}

This paper describes surface area measurements based on local estimates of isosurfaces originating from a marching cubes representation. We show how improved precision and accuracy can be obtained by optimizing the area contribution for one of the cases in this representation. Our results indicate improved 

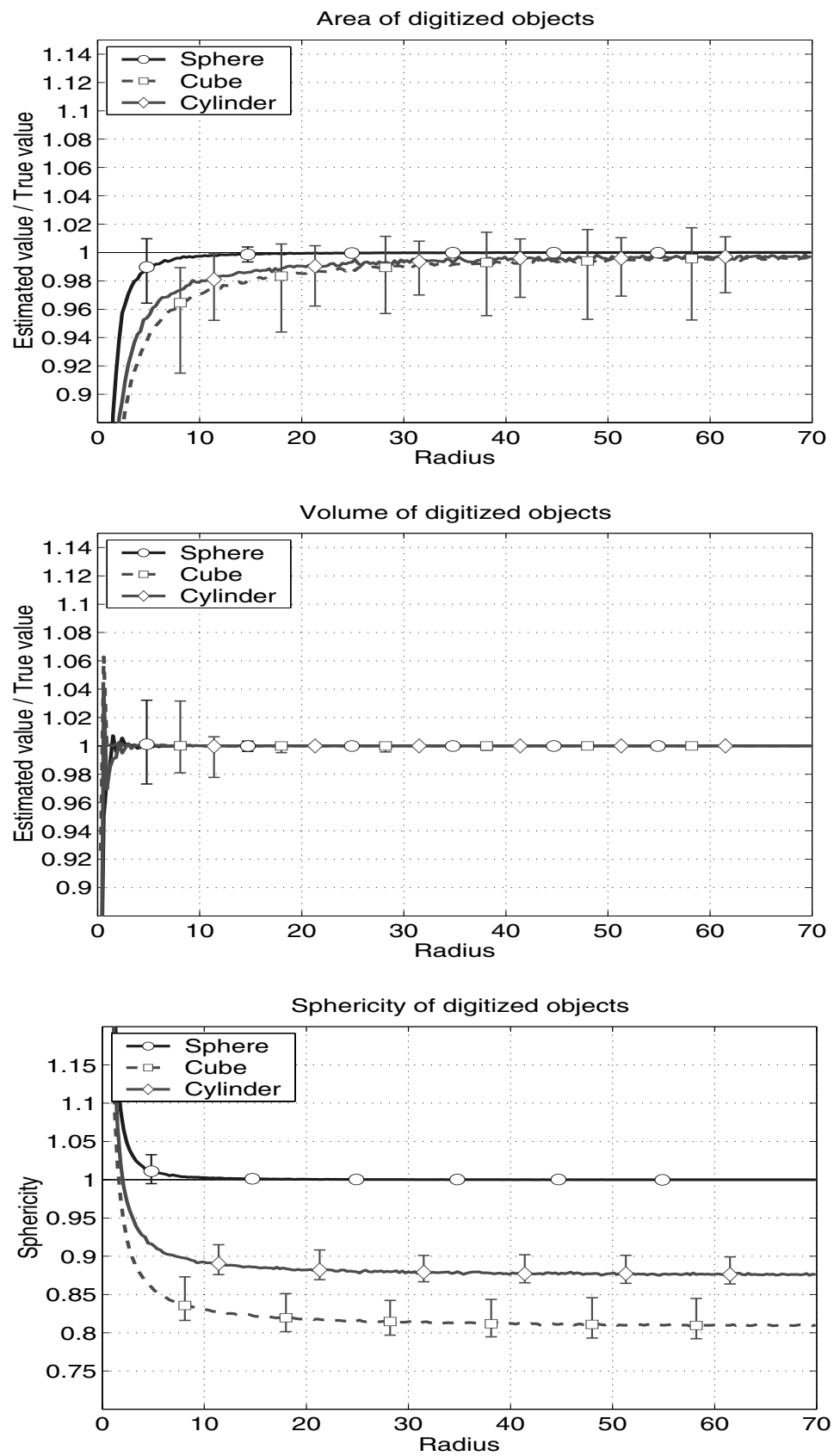

Fig. 9. Measurements for digitized spheres, cubes, and cylinders of increasing size. Top: Surface area estimates divided by the true surface area. Middle: Volume estimates divided by the true volume. Bottom: Sphericity estimates. 
robustness when measuring compactness, or sphericity, to distinguish classes of objects. Non-convex and more natural shapes should be studied in further work.

Another future challenge is to analyze all of the $m$-cube configurations and assign proper weights to them in order to obtain accurate measurements with high precision. This would be an optimization process in 13 dimensions; there are 13 cases of area contributions.

Our surface area computations are performed for a binary marching cubes representation, where the isosurface intersects the marching cube midway between the corners. Further studies on a grey-level marching cubes representation, where the isosurface is approximated according to some interpolation of the grey-level values of the boundary voxels, seems worth pursuing after the promising results for the binary case.

Acknowledgements. We thank Prof. Gunilla Borgefors, Centre for Image Analysis, Uppsala, Sweden, and Prof. Jayaram K. Udupa, Medical Image Processing Group, University of Pennsylvania, Philadelphia, for helpful comments on our work.

\section{References}

1. G. Borgefors. Distance transformations in digital images. Computer Vision, Graphics, and Image Processing, 34:344-371, 1986.

2. S.-s. Chern. Studies in Global Geometry and Analysis, volume 4 of Studies in Mathematics, pages 25-29. The Mathematical Association of America, Washington, DC, 1967.

3. L. Dorst and A. W. M. Smeulders. Length estimators for digitized contours. Computer Vision, Graphics and Image Processing, 40:311-333, 1987.

4. M. J. Dürst. Letters: Additional reference to "Marching Cubes". In Proceedings of ACM SIGGRAPH on Computer Graphics, volume 22(2), pages 72-73, Apr. 1988.

5. H. Freeman. Boundary encoding and processing. In B. S. Lipkin and A. Rosenfeld, editors, Picture Processing and Psychopictorics, pages 241-266. Academic Press, 1970.

6. F. C. A. Groen and P. W. Verbeek. Freeman code probabilities of object boundary quantized contours. Computer Graphics and Image Processing, 7:391-402, 1978.

7. Y. Kenmochi and R. Klette. Surface area estimation for digitized regular solids. In L. J. Latecki, R. A. Melter, D. M. Mount, and A. Y. Wu, editors, Vision Geometry IX, pages 100-111. Proc. SPIE 4117, 2000.

8. Y. Kenmochi, K. Kotani, and A. Imiya. Marching cubes method with connectivity. In Proc. of IEEE Int. Conference on Image Processing (ICIP'99), pages 361-365, 1999.

9. R. Klette and H. J. Sun. Digital planar segment based polyhedrization for surface area estimation. In C. Arcelli, L. P. Cordella, and G. Sanniti di Baja, editors, Visual Form 2001, volume 2059 of Lecture Notes in Computer Science, pages 356366. Springer-Verlag, 2001.

10. J.-O. Lachaud and A. Montanvert. Digital surfaces as a basis for building isosurfaces. In Proc. of 5th IEEE Int. Conference on Image Processing (ICIP'98), volume 2, pages 977-981, Chicago, IL, 1998. 
11. J.-O. Lachaud and A. Montanvert. Continuous analogs of digital boundaries: A topological approach to iso-surfaces. Graphical Models, 62:129-164, 2000.

12. J. Lindblad. Perimeter and area estimates for digitized objects. In Proceedings of SSAB (Swedish Society for Automated Image Analysis) Symposium on Image Analysis, pages 113-117, Norrköping, Sweden, Mar. 2001. Available from the author.

13. W. E. Lorensen and H. E. Cline. Marching Cubes: A high resolution 3D surface construction algorithm. In Proceedings of the 14th ACM SIGGRAPH on Computer Graphics, volume 21(4), pages 163-169, July 1987.

14. I. Nyström, J. K. Udupa, G. J. Grevera, and B. E. Hirsch. Area of and volume enclosed by digital and triangulated surfaces. In S. K. Mun, editor, Medical Imaging 2002: Visualization, Image-Guided Procedures, and Display. Proc. SPIE 4681. Accepted for publication.

15. J. K. Udupa. Multidimensional digital boundaries. Graphical Models and Image Processing, 56(4):311-323, July 1994.

16. A. Van Gelder and J. Wilhelms. Topological considerations in isosurface generation. ACM Transactions on Graphics, 13(4):337-375, 1994.

17. A. M. Vossepoel and A. W. M. Smeulders. Vector code probability and metrication error in the representation of straight lines of finite length. Computer Graphics and Image Processing, 20:347-364, 1982. 